\title{
Luchas por la tierra, contradicciones sociales y sistema político. El caso de las zonas ejidales y comunales en la ciudad de México (1980-1984)*
}

\author{
Julio Calderón Cockburn
}

En este trabajo se presentan los resultados de una investigación que, apoyada básicamente en el análisis de los periódicos de la época, en entrevistas a lideres de colonias populares y en la consulta de la bibliografía disponible, describe las características más relevantes de las luchas por la tierra, en zonas ejidales y comunales del área metropolitana de la ciudad de México, entre 1980 y 1984.

Después de hacer referencia al contexto general dentro del que se dan estas luchas (particularmente cómo se ha producido el crecimiento urbano sobre tierras colectivas, y sobre todo en aquellas que fueron entregadas a los campesinos con la reforma agraria), el articulo se centra en el análisis de las contradicciones sociales producto de las ventas ilegales de esas tierras. Se hace referencia, a partir del análisis periodístico, a los sujetos movilizados en las luchas, a su relación con los objetivos de la movilización, y a las oposiciones generadas en cada uno de los casos estudiados. Esto permite al autor ofrecer una visión (para la zona y el periodo analizados) del número y tipo de luchas ocurridas, de las características de los protagonistas y de los motivos por los cuales se movilizan.

El trabajo termina con una interpretación de estas luchas a la luz de una breve caracterización del sistema político mexicano, retomando al mismo tiempo algunos elementos conceptuales presentados por investigadores sociales que han teorizado sobre el tema de los movimientos sociales.

Se concluye que, si bien los conflictos sociales analizados no han afectado el sistema de dominación en México, se han dado importantes luchas politizadas que alteraron los canales tradicionales de participación ciudadana.

\section{Introduccion}

El objetivo de este artículo es analizar las luchas por la tierra ocurridas entre 1980 y 1984 en las zonas ejidales y comunales del área metropolitana de la ciudad de México (AMCM). Entendemos por "luchas por la tierra" aquellas acciones colectivas protagonizadas tanto por miembros de los núcleos agrarios como por colonos, y que tienen básicamente por finalidad defender las tierras para evitar su transformacion a usos urbanos y asegurar la permanencia legal de los colonos en esas zonas, cuando ellas ya se encuentran incorporadas a la mancha urbana.

La importancia de estudiar las acciones colectivas en las zonas ejidales

* Este articulo es una síntesis de la tesis de maestría Luchas por la tierra en la ciudad de México (1980-1984). El caso de las zonas ejidales y comunales presentada a la Facultad Latinoamericana de Ciencias Sociales (Flacso) y que será publicada por esa institución. Quiero agradecer a Martha Schteingart tanto haber dirigido la tesis como estimularme a que escribiera este articulo. 
y comunales proviene de la constatación, como se verá más adelante, de que han sido mucho más frecuentes en ese tipo de tierras que en las de propiedad privada o estatal. Esta preponderancia es consecuencia del peso que tienen las tierras colectivas en las áreas de expansión de la metrópoli.

El artículo está dividido en tres partes. En la primera se presentan algunos aspectos básicos de las situaciones que dan origen a las acciones colectivas, tratando de vincular las necesidades de suelo como soporte de actividades urbanas, con las ventas ilegales de tierras ejidales y comunales. En la segunda parte se detectan las contradicciones sociales que se dan entre los agentes enfrentados y sus respectivos intereses. Por último, en la tercera parte se analizan los efectos de estas acciones colectivas en el sistema político mexicano.

Reforma agraria, desarrollo urbano y ventas ilegales de tierras

En 1915, como producto de la Revolución mexicana, se inició un proceso de reforma agraria que consistió en expropiar las grandes haciendas y otorgar las tierras a campesinos organizados en ejidos.

Como consecuencia, la estructura de la tenencia de la tierra en la parte no urbanizada del Distrito Federal y en los municipios del estado de México que hoy forman parte del AMCM (o son vecinos a esa área), sufrió una fuerte modificación: las tierras ejidales y comunales, hacia fines de la década de los treinta, representaban ya la mayor parte de la superficie no ocupada por la mancha urbana, en esas unidades político-administrativas.

Si se considera el aporte de los diferentes tipos de tenencia a la expansión de la ciudad de México, se tiene lo siguiente: entre 1940 y 1975 la mancha urbana creció en $675.1 \mathrm{~km}^{2} ; \mathbf{4 8} \%$ de ese crecimiento se produjo sobre tierras ejidales y comunales, $41.6 \%$ sobre tierras privadas y $10.4 \%$ sobre terrenos de propiedad del Estado. Esto significa que la expansión urbana se ha producido en mayor medida sobre tierras de las comunidades agrarias (Schteingart, 1983).

Tal constatación empírica requiere de una explicación. No deja de sorprender este hecho, si se considera que la legislación había declarado que las tierras ejidales y comunales ${ }^{1}$ no podían ser comercializadas para usos urbanos o agrícolas, pues de acuerdo con la ley son inalienables e imprescriptibles. Los mecanismos por los que esas tierras pasaron a usos urbanos fueron tanto legales como ilegales.

\footnotetext{
${ }^{1}$ Mientras que las tierras ejidales fueron otorgadas por la reforma agraria, las comunales remontan sus derechos a la época de la Corona española. Pero en ambos casos está prohibida la comercialización.
} 
Los mecanismos legales asumieron dos formas: las expropiaciones a cargo del aparato del Estado y las permutas a favor de fraccionadores privados.

Las expropiaciones autorizadas en función de utilidad pública y social, consistían en que el Estado expropiaba los terrenos, indemnizaba a los ejidatarios y los destinaba para la localización de parques industriales, equipamientos colectivos (obras viales, escuelas, etc.) y programas públicos de vivienda. Entre 1940 y 1976 el Estado expropió sólo en el Distrito Federal unas 4971.35 ha, que destinó para parques industriales (1 926 ha), viviendas (1 405 ha) y equipamientos (1 640 ha) (Cruz, 1981). Por el uso urbano adjudicado a esas tierras, puede decirse que el Estado los adecuó a las necesidades del proceso de urbanización capitalista, fortalecido a partir de los años cuarenta.

Las permutas permitieron de hecho que a partir de ciertas compensaciones (en tierra y/o en dinero), los ejidatarios aceptaran dejar sus tierras, que pasaron a manos de promotores para la creación, sobre todo, de fraccionamientos residenciales. Entre 1940 y 1976, unas 3982 ha ejidales pasaron en el Distrito Federal a usos urbanos bajo esta modalidad. Mediante la permuta, el capital inmobiliario pudo liberar para un uso urbano residencial las tierras colectivas que no podían venderse o expropiarse para esos usos.

El mecanismo ilegal consistió en la venta de tierras colectivas a sectores medios o populares por parte de los comisariados ejidales o de los ejidatarios en su conjunto. Pese a ser una transacción prohibida y carente de valor legal, entre 1940 y 1976 unas 6585 ha se incorporaron en el Distrito Federal a un uso urbano por esta vía (Cruz, 1981).

Nos detendremos en el análisis de los procesos de ventas ilegales porque ellos han servido de base para la creación de situaciones que han originado los conflictos por la tierra en este tipo de tenencia.

Antes de la Revolución mexicana, los conflictos que se daban por la tierra enfrentaban a los pueblos y las comunidades campesinas con los terratenientes despojadores. Esta contradicción social fue precisamente uno de los detonantes de la Revolución. Con el triunfo del proceso armado, la reforma agraria dotó de tierras a los campesinos mediante la constitución de ejidos, frenando un incipiente proceso de proletarización.

Pero la reforma agraria fue un proceso difícil por las presiones de los terratenientes y de los campesinos; la dotación de tierras no fue suficiente para solucionar la subsistencia de las familias campesinas. Surgieron otros problemas que a la postre se convertirían en factores endógenos que impulsaron las ventas ilegales. Entre ellos pueden mencionarse:

a) la cantidad de tierras entregadas a los campesinos fue escasa. En 1930 se calculaba que en los ejidos del Distrito Federal, a cada beneficiario le había correspondido un promedio de 1.2 ha (Simpson, 1937);

b) la calidad de las tierras entregadas no fue adecuada. En la medida 
que el proceso de reforma agraria permitió a los hacendados decidir qué parte de los terrenos conservaban (hasta $150 \mathrm{ha}$ ), ellos optaron por las mejores, dejando a los ejidatarios tierras de temporal, cuando no salitrosas, boscosas o pedregales, y

c) los ejidatarios no obtuvieron apoyo crediticio ni tampoco técnico para solucionar el problema de la escasez de agua. En 1956, por ejemplo, el Banco Ejidal sólo hacía préstamos a predios que superaran las cinco ha, y ni siquiera tenía una sucursal en el Distrito Federal. Asimismo, la expansión urbana fue consumiendo la disponibilidad del agua subterránea, restando recursos para el cultivo.

Estos problemas contribuyeron decisivamente a perjudicar la viabilidad de la forma productiva en los ejidos, sumiendo a los ejidatarios en una pauperización creciente. Este resultado, según los estudiosos del tema, fue producto de una política agraria que consideraba a la producción en ejidos secundaria frente a la producción agrícola basada en relaciones capitalistas (Gutelman, 1979; Montaño, 1984).

Los ejidatarios, como solución alternativa, empezaron a solicitar al Estado la ampliación de los ejidos. Sin embargo, las autoridades, quizá con la excepción de las del régimen de Cárdenas, no estuvieron muy dispuestas a conceder ampliaciones. Incluso se estableció que sólo podría volverse a solicitar una ampliación pasados 10 años después de una negativa.

Mientras que los factores endógenos generaban una crisis económica en los ejidos, desde los años cuarenta empezó a desarrollarse en México la industrialización por sustitución de importaciones. Este desarrollo se centró principalmente en la ciudad de México, creando una demanda de suelo para instalar fábricas, viviendas, oficinas, equipamientos, etc. La solución a esta necesidad se veía frenada, como se ha visto, porque gran parte de las áreas de expansión urbana se encontraba en manos de los ejidatarios.

Este enfrentamiento entre las lógicas de dos formas productivas en torno al suelo (como soporte para un uso urbano o para cultivar), produciría un conjunto de situaciones que también permiten explicar las ventas ilegales. Entre éstas, pueden mencionarse las siguientes:

a) la reducción de las áreas de posesión colectiva por efecto de las expropiaciones y permutas. Si ya las tierras asignadas a los ejidatarios por la reforma agraria eran insuficientes, no es difícil imaginar las consecuencias de un mayor cercenamiento (aceptado a regañadientes) por la acción estatal y de las inmobiliarias privadas;

b) la expansión urbana de la ciudad de México, acompañada de las obras de infraestructura, elevó los precios del suelo de las áreas de tenencia colectiva. Esta elevación llevó a que los ejidatarios ubicados en las fronteras de la expansión obtuvieran más ingresos vendiendo la tierra que cultivándola; 
c) el gran crecimiento de una población trabajadora de bajos ingresos generó una gran demanda de tierras y en particular una demanda "informal" de la tierra ejidal, y

d) las nuevas fuentes de empleo vinculadas al desarrollo capitalista no sólo fueron absorbidas por personas recién migradas, sino que los propios ejidatarios, ante su pauperización, se incorporaron a las actividades urbanas proletarizándose. Los ingresos percibidos como proletarios fueron seguramente mayores que aquellos a los que podrían haber accedido como cultivadores.

Este conjunto de condiciones materiales, producto de la destrucción de la economía ejidal y del avance del capitalismo industrial, impulsó una modificación en la relación social de los ejidatarios en torno a la tierra. Analíticamente es posible destacar dos momentos: en primer lugar, la tierra, anteriormente vista como valor de uso agrícola, pasó a interesar como valor de uso urbano, es decir, como lugar de habitación; este momento se expresa en las demandas, en los años cincuenta, para la constitución de zonas urbano-ejidales. En segundo lugar, la tierra dejó de ser considerada como valor de uso para convertirse en mercancía, es decir, en un valor de cambio que aporta una renta del suelo. Este momento se ubica en los años sesenta y, particularmente, en los setenta.

La relación entre las ventas ilegales de las tierras colectivas y la sociedad global es diversa. Entre esas concatenaciones puede señalarse el hecho de que la mercantilización de la tierra, como históricamente se ha demostrado, es un fenómeno que responde a sociedades en que predomina el capitalismo (Rossi, 1982; Topalov, 1979). Asimismo, la venta ilegal implica la separación del productor de sus medios de producción, y al ejidatario sólo le queda la proletarización o tender hacia la pequeña burguesía. Por último, con la venta ilegal se modifican los agentes involucrados en la relación con la tierra: de una relación contradictoria entre los campesinos y los terratenientes o el Estado se pasa a otra que va a enfrentar a los ejidatarios con los colonos o a los ejidatarios de base con sus comisariados ejidales.

\section{Las contradicciones sociales producto de las ventas ilegales}

El proceso de venta ilegal consiste en que el conjunto de ejidatarios o el comisariado ejidal venden sus posesiones lotificadas a colonos, a precios por debajo de los que existen en el mercado formal del suelo. La transacción inicial no es de por sí conflictiva; el colono pauperizado encuentra aquí una de sus escasas posibilidades de acceder a una de las condiciones de su reproducción social: un terreno sin servicios sobre el que "autoconstruira". De alli que incluso se establezcan entre colonos y fraccionadores clandestinos relaciones de amistad, compadrazgo o colaboración. 
Pero esta modalidad de apropiación es, como se ha dicho, ilegal. El colono carece de escrituración y de servicios; el ejidatario, que ya no cultiva sino que habita, también carece de la seguridad que otorga la propiedad del terreno.

Esta situación intenta ser "saneada" por el aparato estatal mediante el proceso de regularización. Este consiste en que el Estado, a pedido de los colonos o de los ejidatarios, expropia mediante decreto el conjunto de las tierras de una colonia ilegal y asume su propiedad. Luego, se tasa la valorización agrícola comercial de las tierras y se fija una cantidad a ser pagada por los colonos, misma que se distribuye entre el Estado y los miembros de los núcleos agrarios. Como feliz corolario, tanto los colonos como los ejidatarios acceden a la propiedad legal del terreno en que habitan.

Vista desde la óptica estatal, la regularización es poco menos que una panacea para todos. Para los colonos, porque al fin podrán obtener un "disfrute lícito" de sus lotes y podrán comprar los servicios urbanos; para los ejidatarios, porque accederán gratuitamente a sus solares y recibirán una indemnización, y para el Estado, porque obtendrá ingresos por su participación en la regularización y empezará a cobrar impuestos urbanos. Como final feliz, se pensaba que el sistema podría reforzar sus bases de legitimidad ante colonos y ejidatarios (Bejarano, 1983).

Desde la óptica de la realidad social la cuestión es muy distinta, ya que con la regularización hay grupos sociales favorecidos y otros perjudicados. Resultarian favorecidos los ejidatarios y comuneros porque al dinero obtenido a través de la venta ilegal se añadirían más beneficios por concepto de indemnización y obtendrían gratuitamente sus lotes (a veces de grandes dimensiones para uso residencial); los colonos podrían salir perjudicados porque aparte del pago ilegal inicial deberían volver a realizar pagos para cubrir, por ejemplo, la indemnización; además, deberian comenzar a pagar impuestos por asentamientos sin servicios, en los que la valorización de la tierra ha sido producida por ellos mismos, con su esfuerzo.

Paradoja social de las normas jurídicas: el agente que violó la ley, al vender un objeto inalienable, lejos de ser sancionado, es premiado. A quien adquirió, acicateado por su situación de pobreza, la ley le reconoce más deberes que derechos. Esta paradoja jurídica se explica porque la ley declara inexistente el primer pago (Azuela, 1983).

Los conflictos que se desprenden de estas situaciones son diversos: en general, los ejidatarios pugnarán por elevar los montos de indemnización a pagar por los colonos; en otros casos, los ejidatarios denunciarán que las ventas ilegales fueron responsabilidad de sus comisariados; los colonos, por su parte, se negarán a efectuar otro pago o, en su defecto, pugnan por reducir el monto de indemnización.

Para comprender el carácter de las contradicciones sociales, vamos a tomar como referencia la información empírica obtenida de nuestra pro- 
pia investigación. ${ }^{2}$ Primero hay que aclarar que de los 120 casos detectados en ese estudio, $51 \%$ ( 61 casos) se localizan en zonas ejidales o comunales; $23 \%$ en terrenos de propiedad privada, $15 \%$ en zonas en litigio y $11 \%$ en tierras públicas. Como ya dijimos, esto justifica por qué se centra el estudio en las luchas por la tierra en zonas ejidales y comunales. Además, de las 61 acciones colectivas registradas entre 1980 y 1984 unas 26 fueron dirigidas por ejidatarios ( $43 \%$ ) y unas 35 por colonos $(57 \%){ }^{3}$

Para una aproximación general partiremos del cuadro 1, que ofrece la relación entre los sujetos movilizados y los objetivos de esa movilización. En el caso de los colonos, predomina el objetivo de "contra próximo desalojo" con 17 casos ( $48 \%$ ), seguido de "obtención de la propiedad y regularización" con ocho casos (23\%), "restitución de tierras" con seis casos $(17 \%)$ y "otros" (12\%). En cambio, los objetivos de los ejidatarios y comuneros como sujetos varían significativamente: predomina "contra venta de tierras ejidales y comunales" con 14 casos (54\%), seguido por "contra expropiaciones" con ocho casos (31\%) y "otros" (15\%). A partir de esta aproximación puede afirmarse que se trata de acciones colectivas con dinámicas diferenciadas.

Entre los colonos predomina la defensa de la tierra como valor de uso, para habitarla, contra los intereses de los adversarios que intentan despojarlos. Un indicador de este fenómeno es el predominio de las luchas contra el desalojo. La presencia de "restitución de tierras" significa que el desalojo se ha consumado y los colonos exigen volver a habitar los mismos terrenos. El objetivo de "propiedad y regularización" se explica ante situaciones en las que, si bien no existe una inmediata amenaza de desalojo, los colonos quieren asegurar una permanencia legal en la zona. Resulta claro, pues, que los objetivos de los colonos son pugnar por la tierra como valor de uso para asegurar sus condiciones de reproducción social, mismas que, en términos de vivienda, no pueden ser cubiertas por los programas públicos y, mucho menos, por la acción de los promotores inmobiliarios privados.

Entre los ejidatarios predomina la defensa de la tierra contra procesos diferentes: en unos casos contra los intentos de los comisariados ejidales por venderla ilegalmente (51\%); en otros, contra el aparato del Estado, que quiere expropiar las tierras (31\%). En términos de lo que la información periodística - que es nuestra fuente- permite extraer, el interés de los ejidatarios es mantener las tierras de cultivo frente a agentes interesados en comercializarlas o en destinarlas para un uso urbano. Se requeriría realizar estudios de caso concretos para deslindar si su interés es cultivar o reservar las tierras para luego comercializarlas ellos mismos.

\footnotetext{
${ }^{2}$ Esta información procede de la aplicación de un código hemerográfico a la lectura de periódicos (El Día, Excélsior, UnomásUno, La Jornada) aparecidos entre 1980 y 1984.

${ }^{3} \mathrm{Se}$ incluyen aqui tres casos que movilizaron conjuntamente a colonos y ejidatarios de base porque la dirección correspondió a los primeros.
} 


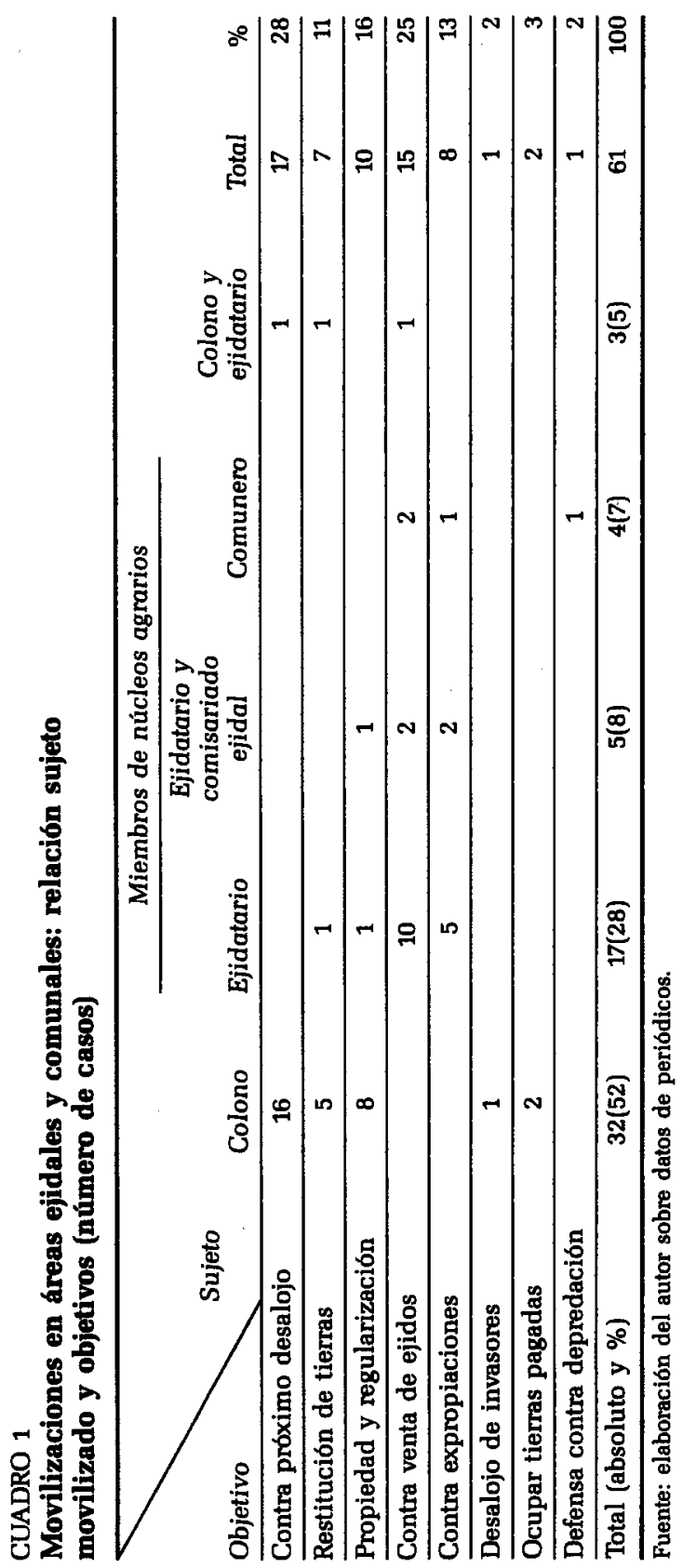


En el cuadro 2 se observa a los sujetos y los adversarios enfrentados en los conflictos. Los colonos (solos o junto con ejidatarios) se han enfrentado a los comisariados ejidales en 14 casos $(40 \%)$, a los fraccionadores privados en nueve casos ( $26 \%$ ), al gobierno en seis casos (17\%), al conjunto de los ejidatarios en cinco casos $(14 \%)$ y a un "coyote" en un caso $(3 \%)$. Por su parte, los miembros de los núcleos agrarios (ejidatarios más comisariados y comuneros) se han enfrentado a los comisariados ejidales (vigentes o anteriores) en 11 casos ( $42 \%$ ), al gobierno en siete casos $(27 \%)$, a fraccionadores privados en cinco casos $(19 \%)$, a empresas privadas en dos casos ( $8 \%$ ) y a colonos en un caso (4 por ciento).

Llama la atención que mientras que las luchas de colonos y ejidatarios se distinguen a nivel de objetivos, a nivel de adversarios se asimilan. En efecto, los colonos cuestionan a los comisariados en $40 \%$ de los casos, mientras los ejidatarios lo hacen en $42 \%$. Es decir, que hay distintos sujetos, distintos objetivos pero un mismo adversario. Para precisar este aspecto fue necesario efectuar un cruce entre los adversarios y sus objetivos, cuadro que no incluimos en esta versión (Calderón, 1986), y que muestra que en los 23 casos en que se impugnó a los comisariados la acusación fue la misma: especular con la propiedad colectiva.

A esta altura es necesario explicitar quiénes son estos comisariados tan impugnados. El comisariado ejidal es la autoridad en el ejido; es electo por la asamblea general de ejidatarios cada tres años. Su función es doble: por un lado, administra los bienes comunales y por otro, se vincula al sistema de dominación integrándose a la Confederación Nacional Campesina (CNC), organismo del partido oficial que opera en las zonas rurales. En los procesos de venta ilegal, como lo muestra esta investigación y otros estudios de caso (Durand, 1983; Lugo y Bejarano, 1981), la CNC ha estado a la vanguardia de la especulación con la tierra.

Ahora bien, para profundizar en el carácter de las contradicciones sociales que producen estas acciones colectivas, vamos a detenernos en los diferentes tipos de conflictos.

\section{Los miembros de los núcleos agrarios}

De los 26 casos en que los miembros de los núcleos agrarios (n.a.) se han constituido en sujetos movilizados vamos a establecer cinco subtipos de conflictos, definidos a partir de los adversarios.

Subtipo 1: miembros de n.a. contra miembros de n.a. (11 casos)

Estas acciones colectivas han sido dirigidas por los ejidatarios contra sus

\footnotetext{
4 "Coyote" es el agente que organiza invasiones y cobra a los colonos por la permanencia en la zona, mientras que negocia una "transacción" con el gobierno y los propietarios.
} 


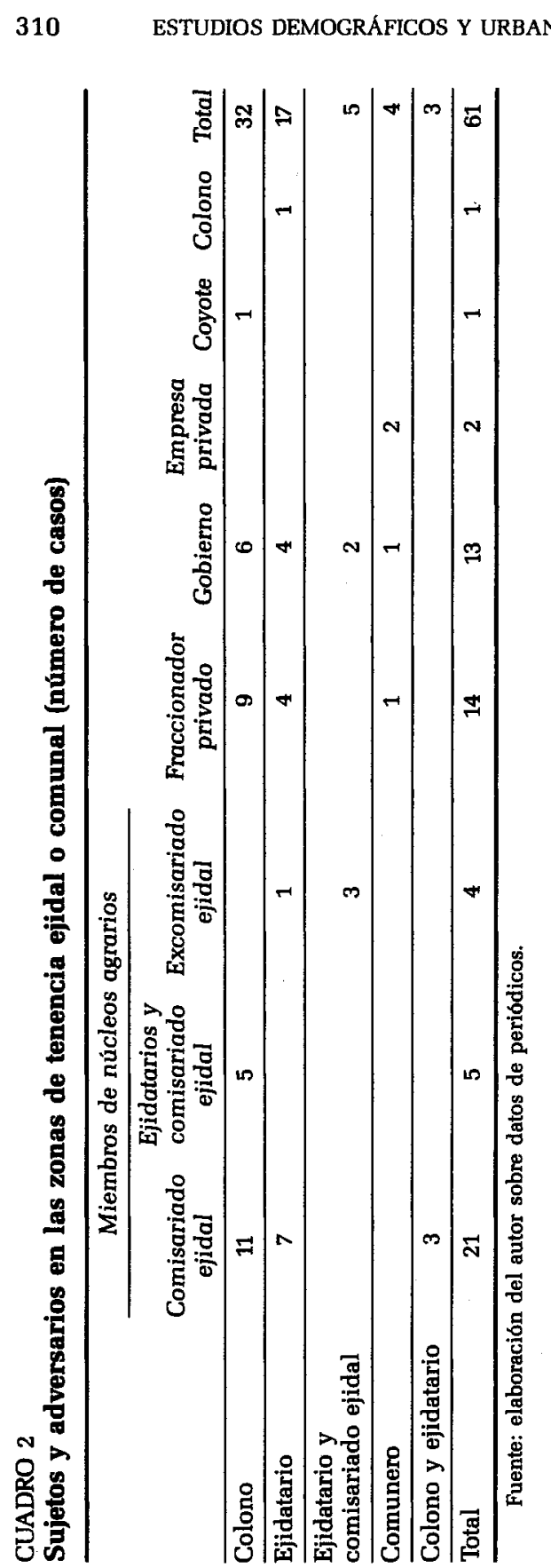


comisariados ejidales en protesta por la venta ilegal de tierras ejidales. En este subtipo se pueden presentar dos situaciones. En unos casos, se trata de ejidatarios que aún cultivan la tierra y defienden su posesión contra comisariados y autoridades, que buscan especular con ella. Como es lógico, estos conflictos han ocurrido en los municipios conurbados del estado de México o en delegaciones del Distrito Federal que no se encuentran totalmente urbanizadas. Entre estos casos se tienen los de Tlazala de Fabela y Francisco Chimalpa en Naucalpan, Sebastián Chimalpa en los Reyes la Páz, San Vicente Chicoloapan en Texcoco y Tepepan en Xochimilco.

En otros casos se trata de ejidatarios que ya no cultivan la tierra, pero que reclaman su participación en los montos de indemnización por concepto de regularización. Son los casos de San Jerónimo Lídice en Magdalena Contreras o Cuautepec Barrio Alto en Madero.

Subtipo 2: miembros de n.a. contra el aparato estatal (siete casos)

Se trata de acciones dirigidas por comuneros o ejidatarios contra el aparato estatal, fundamentalmente con el objetivo de oponerse a la expropiación de sus tierras. Estos casos incluyen a los ejidatarios de San Juan de Aragón, a los del pueblo de Xochimilco, a los de San Francisco de Tlalnepantla y de Santa María de Cuautepec, así como a los chinamperos de Iztapalapa. En este último caso, la lucha fue inicialmente contra la expropiación de sus terrenos para construir una central de abastos; luego de la expropiación, la lucha derivó en la exigencia de un mayor monto de indemnización.

Subtipo 3: miembros de n.a. contra fraccionadores privados (cinco casos)

Han sido conflictos en que los miembros de n.a. se han enfrentado a fraccionadoras privadas que habían ocupado tierras comunales. Son los casos de las luchas del pueblo de Magdalena Contreras, de El Mirador de Huixquilucan, de San Miguel Ajusco, de San Lucas de Tepletalco y de Santa María de Tulpetlac.

Subtipo 4: miembros de n.a. contra empresas privadas (dos casos)

En estas acciones, los miembros de n.a. se han opuesto a que las empresas privadas se apropien de sus bosques con el objetivo de obtener materias primas. Son los casos de los comuneros de San Mateo Tlaltenango (Cuajimalpa) y San Lorenzo de Tlacoyucan (Milpa Alta) contra la papelera Loreto y Peña Pobre y Champiñones el Encino.

Subtipo 5: miembros de n.a. contra colonos (un caso)

Este caso se observó en San Jerónimo de Acazulco, donde los ejidatarios 
denunciaron que los colonos querían suplantarlos en el cobro de una indemnización por los terrenos.

A pesar de la diversidad de estas acciones colectivas, es posible reagruparlas analíticamente en torno a dos contradicciones sociales.

En primer lugar, la que opone los intereses de los miembros de n.a. por conservar la tierra como valor de uso (agrícola y habitacional), contra los intereses de aquellos agentes que quieren mercantilizarla. Esto es claro en las luchas que han tenido como adversarios a los comisariados ejidales y a los fraccionadores privados. Una variante de esta contradicción es la lucha contra las empresas privadas en que se opone el valor de uso de la tierra a su incorporación como medio de producción (materias primas) para las empresas.

En segundo lugar, la contradicción en torno a los montos de expropiación o a la expropiación misma opone a los miembros de n.a. con el aparato estatal y los colonos. En el caso de la expropiación, los miembros de n.a. buscan canalizar mayores montos monetarios por la tierra, es decir, obtener una especie de renta del suelo. En cuanto a la oposición a las expropiaciones, se opone la defensa del ejidatario al interés del Estado de dar un uso urbano a esos terrenos.

\section{Los colonos como sujetos}

Tembién aquí las luchas dirigidas por los colonos ( 35 casos) se han subdividido en cinco subtipos, de acuerdo con los adversarios enfrentados.

Subtipo 1: colonos contra miembros de n.a. (16 casos)

En estos conflictos se han destacado como objetivos de lucha la defensa contra desalojos (56\%) y la propiedad y regularización (19\%). El predominio de la lucha contra desalojos da cuenta de la violencia de estos conflictos: lo que ha estado en juego es la permanencia en la colonia. Por lo general, los colonos acusan a los ejidatarios (en especial a los comisariados) de haberles vendido ilegalmente los terrenos para luego desconocer la transacción con el objetivo de seguir especulando con la tierra. Estos casos incluyen las luchas de los colonos de la Huizadora, Caracoles, San Nicolás de Chiconautla, Loma Colorada, Bosques de las Lomas, Santa Úrsula Coapa, Héroes de 1910, 2 de Octubre, Cerro del Judío y San Miguel de Teotongo. Por su parte, los miembros de n.a. impugnados han pertenecido a ejidos y comunidades como San Martín de Tepextitlán, San Francisco Tlaltenco, Santo Tomás de Ajusco, San Nicolás de Totolapan, San Bernabé Ocotepec, etcétera. 
Subtipo 2: colonos contra fraccionadores privados (nueve casos)

Estos conflictos responden por lo general a la siguiente situación: los ejidatarios (o sus comisariados) venden la tierra a sectores de altos ingresos quienes, a su vez, las vuelven a vender a los colonos. La situación es compleja, porque son dos procesos de venta ilegal. Los problemas surgen cuando se intenta regularizar la tenencia de la tierra: los colonos exigen su permanencia en la zona porque pagaron por ella; los ejidatarios denuncian que sus comisariados vendieron a sus espaldas y exigen montos de indemnización; los fraccionadores privados demandan que se reconozca su compra ilegal y quieren la indemnización, y los comisariados ejidales niegan la venta ilegal y exigen, a su vez, la indemnización, cuando no el desalojo de los colonos. Algunos casos referidos a este tipo de lucha han sido los de los colonos de Cuchilla de la Magdalena en los Culhuacanes, Paraje las Barracas, Lomas de la Providencia, Aztlán, las Arboledas, Xochiaca, etcétera.

Subtipo 3: colonos contra el gobierno (seis casos)

Este subtipo de conflictos ha ocurrido por lo general cuando los colonos, establecidos en una zona adquirida ilegalmente, son conminados a desalojarla porque el aparato del Estado quiere utilizarla como área verde o parque nacional. Entre los casos más destacados se encuentra el de Bosques del Pedregal y otras colonias del Ajusco que finalmente consiguieron permanecer en la zona. Un caso especial fue el de San José Aculeo, en Iztapalapa, en el que los colonos acusaron al gobierno de querer desalojarlos para ubicar allí a sus seguidores políticos.

Subtipo 4: colonos y ejidatarios contra comisariados (tres casos)

En estos conflictos se unieron los colonos y los ejidatarios para luchar contra los intentos del comisariado ejidal de vender ciertas tierras comunales a fraccionadores privados. Son los casos de Santa Marta del Sur y Santa Úrsula Coapa en Coyoacán y Cuautepec Barrio Alto en Madero.

Subtipo 5: colonos contra "coyote" (un caso)

Es el caso del campamento Francisco Villa en Iztapalapa, donde los colonos invadieron en 1982 tierras ejidales, dirigidos por líderes a los que pagaron por "protección" para permanecer en la zona. En 1983, al tiempo que los líderes huyeron, los colonos fueron desalojados, iniciando luego su lucha por volver a los terrenos.

En el caso de las luchas dirigidas por los colonos, también hay que señalar el carácter de las contradicciones sociales que enfrentan a sujetos y adversarios. 
La contradicción social más importante es la que se da entre los colonos interesados en defender su permanencia en el terreno y los adversarios, que buscan desalojarlos para volver a comercializar la tierra. En otras palabras, los colonos (obreros, trabajadores de servicios, etc.) reclaman la tierra como un valor de uso (para habitarla) y, al oponerse al desalojo o a mayores costos, defienden sus condiciones de reproducción y sus salarios. En cambio, los adversarios, por lo general ejidatarios, pero también fraccionadores privados, pugnan por desalojar a los colonos para volver a vender la tierra a mayores precios o, en su defecto, para elevar los montos por indemnización. En ambos casos su interés es obtener mayores tasas monetarias por concepto de renta del suelo.

Una segunda contradicción se da entre los colonos que requieren la tierra como valor de uso y el aparato estatal que quiere reservar ciertos terrenos para equipamientos. En estos casos, el aparato estatal es interpelado directamente como adversario, mientras que en los casos en que se enfrentan agentes privados, también el aparato estatal resulta cuestionado por apoyar a los comisariados ejidales o a los fraccionadores.

\section{Movimientos sociales y sistema político}

Las contradicciones sociales que se han detectado sólo se explican al interior de una formación social predominantemente capitalista. Es necesario reiterar que la mercantilización del suelo para un uso urbano acorde con las necesidades del crecimiento urbano-industrial, se explica históricamente en los momentos en que se desarrolla el proceso de sustitución de importaciones. En esa medida, la pugna entre la tierra como mercancía o como valor de uso es una contradicción que acompaña la urbanización capitalista. Es evidente entonces que existe una relación entre las contradicciones apuntadas y el sistema social; sin embargo, los efectos que los movimientos surgidos a partir de esas contradicciones producen en el sistema de dominación no son tan claros.

De hecho, se ha tendido a establecer una distinción en los niveles en que las acciones colectivas (por utilizar un término genérico) afectan al sistema político. Se suele entonces distinguir entre "conducta colectiva", "lucha" y "movimiento social" (Touraine, 1984); o entre "acción conflictiva" y "movimiento social" (Melucci, 1979). Ya dentro de la sociología urbana, se distingue a los "movimientos urbanos" de los "movimientos sociales urbanos"; a la "reivindicación" de la "lucha urbana" (Castells, 1976; Della Pergola, 1974; Borja, 1976). El interés de la última parte de este artículo es ver en qué medida las luchas por la tierra reseñadas afectan al sistema político de dominación, lo que lleva a establecer las mediaciones necesarias entre las luchas y su politización.

Para ello vamos a utilizar en el análisis dos términos descriptivos que 
han servido para ordenar la información hemerográfica: los movimientos espontáneos, entendidos como las acciones de corta duración, sin presencia importante de organizaciones sociales extralocales y sin una respuesta integral del sistema político; y los movimientos orgánicos, que tienen una mayor duración, apoyo de organizaciones extralocales y que han enfrentado, en sus niveles de manifestación, al sistema de dominación.

Estos términos descriptivos tienen una utilización pragmática. Conforme se vayan analizando las relaciones entre las acciones colectivas y el sistema político se propondrán las conceptualizaciones pertinentes.

Antes de entrar al análisis de la información, es preciso caracterizar brevemente al sistema político mexicano. Se le considera dotado de una fuerte legitimidad que le ha permitido afrontar en forma estable buena parte del periodo de crecimiento económico. Ello ha sido posible gracias a mecanismos ideológicos (derivados del proceso revolucionario), relativas concesiones en la esfera de la distribución y un aparato institucional corporativo y cooptador, todo dependiente de un sistema político unipartidista (Huntington, 1970). Esta institucionalización ha operado en el control de los colonos mediante líderes locales subordinados al partido oficial, a organizaciones corporativas de rango intermedio como la Federación de Colonias Proletarias (FCP), la Confederación Nacional de Organizaciones Populares (CNOP), y mediante las autoridades delegacionales, municipales y, por supuesto, el propio Partido Revolucionario Institucional (PRI), que orquesta el accionar de todas las instituciones mencionadas. Este proceso, en términos generales, es aceptado por los colonos tanto a nivel de sus actitudes individuales como de sus acciones colectivas (Cornelius, 1982).

\section{Los miembros de los núcleos agrarios como sujetos}

Sostenemos la hipótesis de que las luchas por la tierra dirigidas por los miembros de n.a. han afectado en poca medida al sistema de dominación. Esta conclusión se fundamenta en la lectura del cuadro 3.

En primer lugar, se observa que los conflictos se han traducido en su gran mayoría en movimientos espontáneos de corta duración. ${ }^{5}$ De un total de 26 acciones, unas $22(85 \%)$ fueron de corta duración, contra tres casos de mediana duración y sólo uno de larga duración. Si se pasa del análisis global al de los subtipos se observará que los movimientos más prolongados se han dado cuando los adversarios han sido agentes ajenos a la organización ejidal: en dos casos contra empresas privadas, en uno contra

\footnotetext{
${ }^{5}$ La caracterización de la duración de los movimientos fue asi: se consideró de corta duración cuando se expresaban uno o dos hechos en los diarios; de mediana duración entre tres y nueve hechos y de larga duración diez o más hechos.
} 


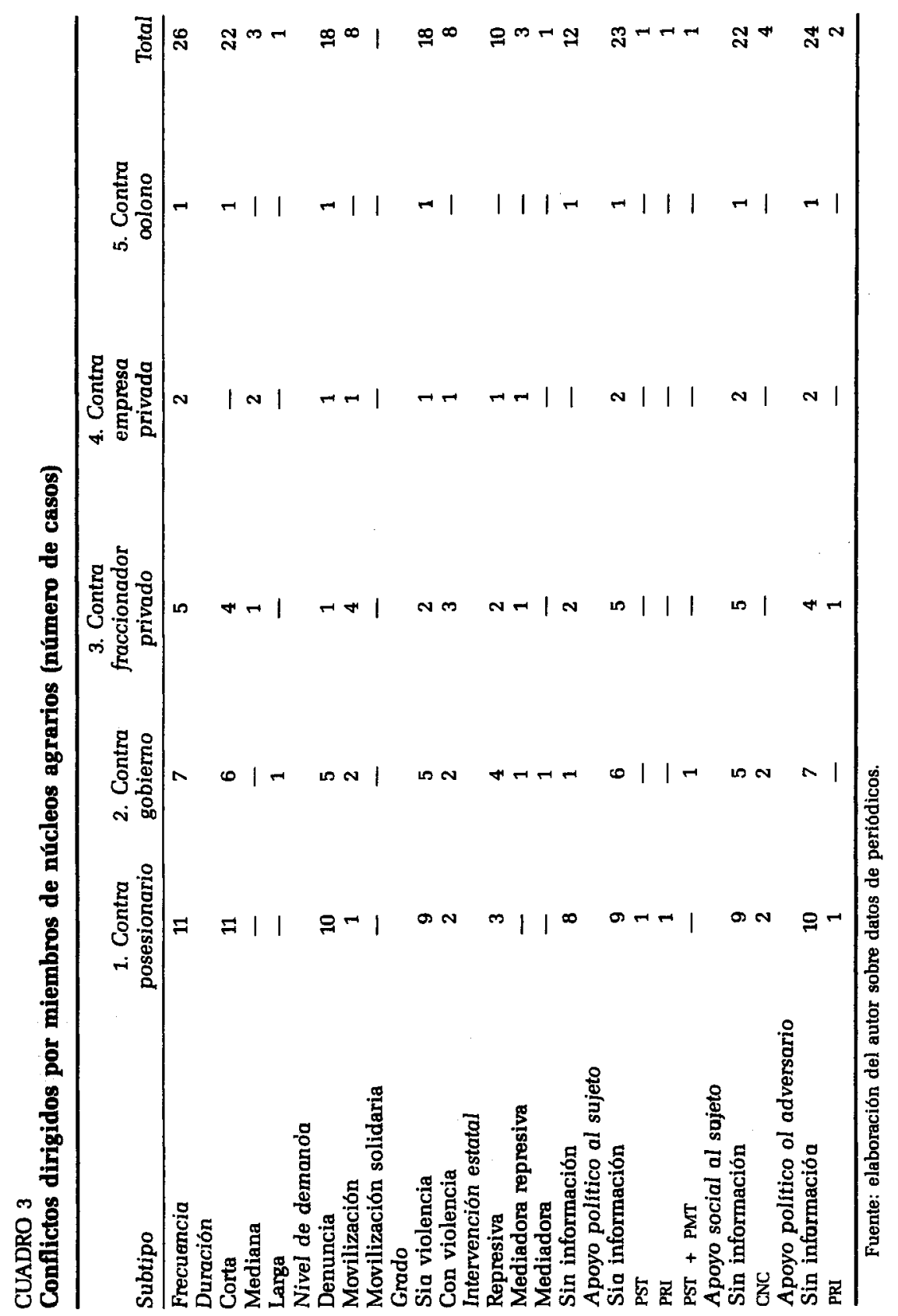


un fraccionador privado (Inmobiliaria Rena) y en otro contra el gobierno.

Es significativo, pues, que aunque las luchas entre los propios miembros de n.a. (ejidatario contra el comisariado, por lo general), han sido las más frecuentes, no han originado movimientos prolongados. Se ha tratado en su mayoría de denuncias formuladas ante la prensa (91\%), de conflictos que no han dado lugar a acciones violentas (82\%) y sin organizaciones extralecales como apoyo u oposición a los sujetos movilizados; en $82 \%$ de los casos no aparecen partidos políticos apoyando al sujeto; en $82 \%$ no existen organizaciones sociales de rango intermedio y en $82 \%$ de los casos no se denunció a ningún partido político vinculado al adversario.

Volviendo al análisis global, con estos mismos indicadores se tiene que de las 26 luchas, en 18 casos (69\%) se trató de denuncias, en 18 casos de acciones no violentas ( $69 \%$ ) y, por parte del aparato estatal, sólo en 10 casos (38\%) se dio un comportamiento represivo. Observando la presencia de organizaciones extralocales, se tiene que en 23 casos $(88 \%)$ no hubo apoyo político al sujeto, en 22 casos (85\%) no hubo apoyo de organizaciones "sociales" al sujeto y que en 24 casos ( $92 \%$ ) no se cuestionó a ninguna organización política apoyando al adversario. Ahora, es importante indicar (información recogida pero que no se encuentra en el cuadro) que en 21 casos ( $81 \%$ ) se acusó a los funcionarios públicos - de las delegaciones, los municipios o la Secretaría de Reforma Agraria- de estar en complicidad con los adversarios. Esto da a entender que a pesar de su carácter espontáneo y poco politizado, estas acciones han revelado ciertos niveles de insatisfacción con el aparato administrativo.

A nuestro criterio, no resulta fácil interpretar estos datos a la luz de un contexto teórico. Siguiendo a Melucci, ${ }^{6}$ podría hablarse de acciones conflictivas en la medida en que se dan entre los límites del sistema. Sin embargo, es difícil establecer si son meramente acciones reivindicativas o si son políticas en el sentido de que utilizan los procesos de decisión del sistema político. La fuente de nuestra información - los periódicospuede no recoger un conjunto de mecanismos subterráneos, de "padrinazgos políticos" y de pugnas al interior del sistema unipartidario. Una mayor y mejor caracterización requiere, sin lugar a dudas, de estudios de caso en profundidad que superen las limitaciones del análisis hemerográfico. ${ }^{7}$

No obstante, a título provisional pueden sugerirse dos hipótesis que expliquen la baja politización de las acciones dirigidas por los ejidatarios

${ }^{6}$ En mi opinión, Melucci es quien más ha avanzado en establecer mediaciones para el análisis de los movimientos sociales y las acciones colectivas. Sin embargo, este reconocimiento no implica aceptar su teorización general, influida por la sociología de la acción de Touraine, en el sentido de que se está en sociedades ya no clasistas y en las que las contradicciones de clase han desaparecido. Ciertamente, recoger aportes de otros paradigmas plantea la necesidad de traducir y reificar esos aportes. Señalo este problema que aqui no puede desarrollarse.

${ }^{7}$ Pero también el análisis hemerográfico tiene sus virtudes: permite aproximaciones generales imposibles de realizar desde los estudios de caso. 
(baja politización en el sentido de que no transgreden las normas del sistema de dominación, ya que acabamos de aclarar que en estas acciones pueden estar presentes organismos políticos que no figuran en la información periodística):

En primer lugar, existe un desajuste entre movimiento y organización. Los movimientos o acciones colectivas, o parten de una organización o acaban constituyéndola. La organización local de los movilizados es un factor clave para entender la dinámica de los conflictos y su prolongación. En estos casos, el desajuste se produce porque los movilizados impugnan, por lo general, a los propios dirigentes de la organización (el comisariado ejidal). No existen canales alternativos de organización para que los ejidatarios impugnen a sus comisariados. La posibilidad de un cambio de liderazgo resulta entorpecido porque, tal como ellos denuncian, la administración estatal está coludida con los comisariados en la especulación de terrenos y nada hace pensar que aceptarán reconocer a líderes de los movilizados. En este sentido, no deja de ser sintomático que los enfrentamientos con agentes externos al ejido sean más prolongados en la medida que hay un mayor ajuste movilizados-organización.

En segundo lugar, se da un parcial desajuste entre la acción colectiva y la base social que dificulta la solidez del movimiento. Hay desajuste cuando la base social de los ejidatarios ya no cultiva la tierra. Más que un grupo, constituyen una masa de individuos unidos por su estatus jurídico y su interés en recibir indemnizaciones, con lo que su identidad resulta mellada por su dispersión ocupacional, existencia de diversos grupos de referencia, etc. Esta explicación es parcial porque la baja politización también se advierte en las acciones de los ejidatarios que aún cultivan la tierra. Para explicar estos casos podría manejarse la hipótesis de que el peso del sistema de dominación, las expectativas de mejoras que de repente se vinculan más a la aceptación del sistema que a su oposición, y la posibilidad de que los ejidatarios que aún cultivan lleguen a transformarse en fraccionadores clandestinos, podrían atentar contra su unidad a mediano o largo plazo.

\section{Los colonos como sujetos}

Los movimientos dirigidos por los colonos han sido relativamente más politizados que los de los ejidatarios. Decimos relativamente porque de los 35 conflictos registrados, en $21(60 \%)$ han predominado los movimientos :spontáneos. Sin embargo, recordemos que en el caso de los ejidatarios la espontaneidad estaba presente en 85 por ciento.

Observando el cuadro 4 vamos a señalar los indicadores de esta relativa politización comparándolos con los resultados obtenidos en el caso de las acciones de los miembros de n.a. 


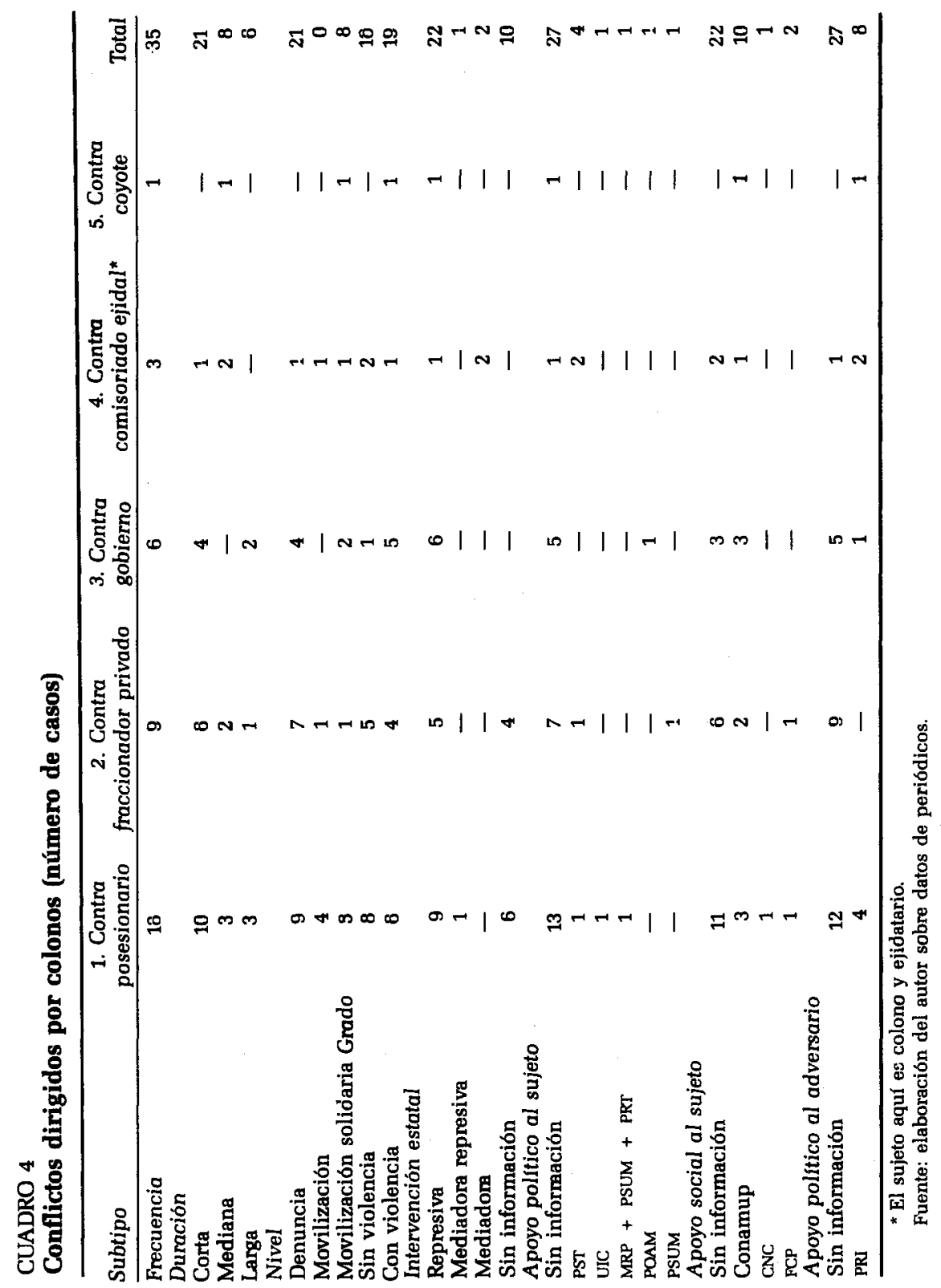


Los movimientos de los colonos se han dado en $60 \%$ a nivel de denuncia, en $17 \%$ a nivel de movilización y en $23 \%$ a nivel de movilización solidaria. Bajo este último término se comprenden aquellas acciones en las que los colonos se han vinculado con otras organizaciones para desarrollar luchas conjuntas. En el caso de los ejidatarios, $69 \%$ de luchas fueron a nivel de denuncia, $31 \%$ a nivel de movilización y ninguna como movilización solidaria.

Con respecto al grado de conflicto, las acciones de los colonos implicaron en $54 \%$ el uso de la violencia, mientras que en la de los ejidatarios sólo se llega a $31 \%$. El aparato estatal tuvo un comportamiento represivo en $68 \%$ de los conflictos dirigidos por colonos, mientras que contra los de los ejidatarios esta actitud se dio en $38 \%$. Esto quiere decir que el aparato estatal ha sido menos tolerante frente a los primeros que frente a los segundos.

Dados los indicadores de mayor presencia de conflictos más prolongados, de movilización, violencia y mayor represión del Estado, es posible encontrar otros indicadores que denoten una oposición política al sistema más intensa, en especial, la presencia de organismos sociales de rango intermedio. Esto se confirma cuando se ve que de los 13 casos (37\%) en que se encuentran organizaciones sociales extralocales, en 10 de ellos está involucrada la Coordinadora Nacional del Movimiento Urbano Popular (Conamup), de tendencia izquierdista, mientras que sólo en tres casos se da la presencia de organizaciones controladas por el PRI (la FCP en dos y la CNC en uno). En cambio, en las luchas de los ejidatarios las organizaciones extralocales se presentaron en cuatro casos (15\%), tratándose en todos ellos de la CNC. No existe, pues, entre los ejidatarios la tendencia a vincularse a organizaciones de oposición.

La presencia directa y explícita de los partidos políticos en estas luchas es baja; en las luchas de los colonos sólo se da en ocho casos $(22 \%)$. El Partido Socialista de los Trabajadores (PST) participó en cuatro casos y las siguientes organizaciones de izquierda en un caso cada una: Partido Socialista Unificado Mexicano (PSUM), la alianza del PSUM con el Partido Revolucionario de los Trabajadores (PRT), y el Movimiento Revolucionario del Pueblo (MRP) y la Unidad de Izquierda Comunista (UIC), y el Partido Obrero Agrarista Mexicano (POAM). En las luchas de los ejidatarios sólo en tres casos hubo presencia de partidos políticos (11.5\%): el PRI apareció en uno, el PST en otro y la alianza PST con el Partido Mexicano de los Trabajadores (PMT) en el tercero. Esta información debe tomarse nuevamente como relativa debido a la fuente periodística utilizada; por lo general, la presencia de los partidos en las luchas no se hace explícita.

Considerando ahora a los partidos políticos que han sido denunciados como vinculados al adversario, en los movimientos de colonos se señaló al PRI en ocho casos (23\%), mientras que en los de los ejidatarios esto ocurrió sólo en dos casos (8\%). Ningún otro partido ha sido denun- 
ciado como vinculado al adversario, de acuerdo a nuestra fuente de información.

Una vez que se ha demostrado que, comparativamente, las luchas de los colonos son más politizadas que las de los miembros de los n.a., quisiéramos explicar por qué se da la politización. Hemos tenido que seleccionar una muestra para llevar a cabo algunos estudios de caso. Analizando las acciones de los colonos por subtipos, se tiene que en el enfrentamiento contra ejidatarios, las acciones de mediana y larga duración llegan a $38 \%$ de los casos, mientras que en las luchas contra fraccionadores privados o el gobierno, sólo llegan a $33 \%$. Asimismo, el predominio de las movilizaciones se da en $44 \%$ de los casos en la lucha contra ejidatarios, mientras que contra el gobierno ellas aparecen en $33 \%$ y contra los fraccionadores en $22 \%$. Una vez que se detectó la importancia de las luchas contra ejidatarios, se seleccionaron los tres casos más destacados por su duración: el de los colonos de 2 de Octubre contra los ejidatarios de San Nicolás de Totolapan; el de Cerro del Judío contra los ejidatarios de San Bernabé Ocotepec y el de San Miguel de Teotongo contra los comuneros fraccionadores de Los Reyes la Paz.

No es posible presentar en este artículo detalles de casos (véase Calderón, 1986: 112-154); nos limitaremos a subrayar, en términos generales, los factores que explican la politización de los conflictos.

Una situación común inicial de los colonos en los tres barrios es su atomización como grupo y la presencia de dirigencias cooptadas. La atomización proviene del hecho de que los colonos adquirieron individualmente sus lotes. La cooptación da cuenta de la capacidad del sistema para imponer sus dirigencias y de la ilusión de los colonos de pensar que habrá mejoras en el hábitat en la medida en que se está con el sistema.

Luego, mediante un proceso lento, complejo e intermitente que esta síntesis no puede detallar, se va formando la acción colectiva, que consiste en el paso de la necesidad (de seguridad legal, servicios, trazados racionales), a la reivindicación. Se perfila la identidad de los sujetos, el reconocimiento de sus adversarios y el delineamiento de sus objetivos (la regularización).

Este paso obedece a la acción de agentes concientizadores (partidos de izquierda, comunidades cristianas de base, militantes de los movimientos estudiantiles y sindicales independientes) y requiere el remplazo de la dirigencia cooptada.

De esta forma, la preparación de la acción colectiva se encuentra ya teñida de politización; posteriormente, el proceso de negociación con las autoridades, la capacidad de movilización autónoma y las limitaçiones del propio aparato del Estado van llevando la politización a un punto máximo: aquel en el cual los movimientos urbanos rompen con los canales tradicionales de participación política en que se habían encapsulado y plantean su constitución como una fuerza social independiente del siste- 
ma. Algunas características que acompañan estas acciones colectivas son la fortaleza organizativa interna, la ligazón con la Conamup, la puesta en práctica de procedimientos de lucha (plantones, cierre de calles, etc.), que se combinan con la negociación y aceptación de los partidos de izquierda, al extremo que en dos de las colonias ( 2 de Octubre y Cerro del Judío) fueron apoyados generalizadamente en las contiendas electorales.

Estos casos constituyen movimientos sociales politizados en la medida en que estas acciones, a partir de las reivindicaciones, rompen las reglas del juego institucional y buscan transformar los canales de participación política.

Es necesario, antes de pasar a ver la reacción del sistema dominante, dejar claramente establecido que estas afirmaciones se refieren a nuestros casos de estudio y en este sentido constituyen excepciones que de ningún modo pueden generalizarse al comportamiento de todos los colonos. Reforzando esta idea queremos señalar dos cuestiones: en primer lugar, que nuestro estudio se centra en los casos de luchas por la tierra y no en las colonias en las que no se lucha (cuyo examen es importante si se quieren hacer afirmaciones generales); en segundo lugar, que en los casos de esas luchas, hemos constatado el predominio de movimientos espontáneos ( 60 por ciento).

El sistema, como es de esperar, está poco dispuesto a aceptar los movimientos independientes, que se escapan de su control; por consiguiente, intenta socavar su organización mediante la acción de los organismos corporativos (FCP, CNOP), las delegaciones administrativas y el partido oficial. Si fracasa el socavamiento y no se consigue una vuelta a una situación de cooptación, el sistema aún puede apelar a congelar la reivindicación, es decir, a no regularizar la tierra.

Para entender esta relación entre los movimientos sociales y el sistema político hay que ser sumamente cuidadosos. Bertoldt Brecht observaba que si el comportamiento humano parece impredecible no es porque no haya determinaciones sino porque hay demasiadas. ${ }^{8}$ Si bien es parcialmente correcto decir que la presencia de un movimiento independiente es un obstáculo para conseguir una solución favorable del problema planteado, es preciso reconocer también que en la ciudad de México, muchas colonias que han obtenido servicios están dirigidas por organizaciones independientes y se encuentran en mejores condiciones que las cooptadas. Explicar por qué la mayoría de las colonias con más necesidades no rompen con la cooptación y se independizan significa analizar los mecanismos de clientelismo y el peso del sistema de dominación. Esta pretensión escapa con mucho a nuestro objeto de estudio.

Es preciso, pues, reconocer la existencia de factores que explican por qué la regularización no ha procedido en las tres colonias estudiadas. En-

\footnotetext{
${ }^{8}$ Citado por Anderson (1985).
} 
tre ellos, puede mencionarse la complejidad de estos conflictos, cuya solución no depende sólo de la relación colonos-Estado sino que también involucra a los ejidatarios como adversarios y grupo de presión. Éstos, mucho más integrados al sistema de dominación, reivindicarán ante el sistema que son los "hijos legítimos de la Revolución" y el clientelismo tendrá que ponerse en marcha. También es necesario mencionar los procedimientos burocráticos que retrasan la regularización y los recursos de amparo presentados en los juzgados por los miembros de los n.a. Por último, una regularización masiva de la tierra - sabiamente descartada por el sistema - generaría una demanda también masiva por servicios urbanos, que pondría en cuestión tanto la estructura de concentración de ingresos de la sociedad (muchos colonos no podrían pagarlo) como la prioridad de los gastos sociales del Estado en lo que se refiere a las condiciones de reproducción de los trabajadores.

Para concluir este análisis de las luchas politizadas, es necesario indicar que la reacción del sistema, si bien consiguió un reflujo del movimiento (por la congelación de la regularización) no logró, sin embargo, retrotraer las cosas a la situación de cooptación anterior: las dirigencias independientes se mantienen en sus cargos y los colonos siguen teniendo una mala imagen de las instituciones oficiales.

Hemos tratado de mostrar aquí cómo el desarrollo urbano ha generado un conjunto de contradicciones sociales; una de éstas, quizá la más importante, opone los intereses por el suelo como valor de uso (urbano o agrícola) contra los intereses por mercantilizarlo; y si bien estos conflictos no han afectado demasiado las bases del sistema de dominación, han existido casos de luchas politizadas que han alterado los canales tradicionales dentro de los que se encierra comúnmente la participación ciudadana.

\section{Bibliografia}

Anderson, Perry (1985), Teoría, política e historia, Siglo xxI, Madrid.

Azuela, Antonio (1983), "La legislación del suelo urbano. ¿Auge o crisis?", en SIAP, Relación Campo-Ciudad, SIAP, México.

Bejarano, Fernando (1983), "La irregularidad de la tenencia de la tierra en las colonias populares", en Revista Mexicana de Sociología núm. 3.

Borja, Jordi (1975), Movimientos sociales urbanos, SIAP, Buenos Aires.

Castells, Manuel (1976), Movimientos sociales urbanos, Siglo XXI, México.

Cornelius, W. (1980), Los inmigrantes pobres en la ciudad de México y la politica, FCE, México.

Cruz, Soledad (1981), El ejido en la urbanización de la ciudad de México, tesis presentada en la UAM Azcapotzalco, México.

Della Pergola, Giuliano (1974), Diritto alla cittá e lotte urbane, Feltrinelli, Milán.

Eckstein, Susan (1982), El Estado y la pobreza urbana en México, Siglo XxI, México.

Gutelman, Michel (1979), Capitalismo y reforma agraria en México, ERA, México. 
Huntington, Samuel (1970), "Social auu' Institutional Dynamics of Oneparty Systems", en Huntington y Moore, Authoritarian Politics in Modern Society, Basic Books, Nueva York.

Melucci, Alberto (1979), Sistema Politico, partiti e movimento sociali, Feltrinelli, Milán.

Montaño, Cristina (1984), La tierra de Iztapalapa, UAM, México.

Rossi, Aldo (1982), La arquitecturc de la ciudad, Gustavo Gili, Barcelona.

Schteingart, Martha (1983), "La incorporación de la tierra rural de propiedad social a la lógica capitalista del desarrollo urbano: el caso de México", en SIAP, Relación campo-ciudad, México.

Simpson, Eyler (1937), The Ejido: Mexico's way out, North California Press.

Topalov, Christian (1979), La urbanización capitalista, Edicol, México.

Touraine, Alain (1984), Le Retour de l'acteur, Fayard, Paris. 